\title{
Excess centrosomes disrupt vascular lumenization and endothelial cell adherens junctions
}

\author{
Danielle B. Buglak ${ }^{1}$ - Erich J. Kushner ${ }^{2,4} \cdot$ Allison P. Marvin ${ }^{2} \cdot$ Katy L. Davis $^{2} \cdot$ Victoria L. Bautch $^{1,2,3}$
}

Received: 27 November 2019 / Accepted: 8 July 2020 / Published online: 22 July 2020

(c) The Author(s) 2020

\begin{abstract}
Proper blood vessel formation requires coordinated changes in endothelial cell polarity and rearrangement of cell-cell junctions to form a functional lumen. One important regulator of cell polarity is the centrosome, which acts as a microtubule organizing center. Excess centrosomes perturb aspects of endothelial cell polarity linked to migration, but whether centrosome number influences apical-basal polarity and cell-cell junctions is unknown. Here, we show that excess centrosomes alter the apical-basal polarity of endothelial cells in angiogenic sprouts and disrupt endothelial cell-cell adherens junctions. Endothelial cells with excess centrosomes had narrower lumens in a 3D sprouting angiogenesis model, and zebrafish intersegmental vessels had reduced perfusion following centrosome overduplication. These results indicate that endothelial cell centrosome number regulates proper lumenization downstream of effects on apical-basal polarity and cell-cell junctions. Endothelial cells with excess centrosomes are prevalent in tumor vessels, suggesting how centrosomes may contribute to tumor vessel dysfunction.
\end{abstract}

Keywords Endothelial cell $\cdot$ Centrosome $\cdot$ Junctions $\cdot$ Polarity $\cdot$ Lumen $\cdot$ Angiogenesis

\section{Introduction}

Angiogenesis is the sprouting of new blood vessels from pre-existing vessels and is crucial during development, pregnancy, wound healing, and tumorigenesis [1-3]. Endothelial cells (EC) form new sprouts to provide conduits for blood flow and create a functional vascular network. This process requires dynamic rearrangement of endothelial cell-cell junctions and establishment of apical-basal polarity [4-8].

Electronic supplementary material The online version of this article (https://doi.org/10.1007/s10456-020-09737-7) contains supplementary material, which is available to authorized users.

Victoria L. Bautch

bautch@med.unc.edu

1 Curriculum in Cell Biology and Physiology, The University of North Carolina at Chapel Hill, Chapel Hill, NC 27599, USA

2 Department of Biology, The University of North Carolina at Chapel Hill, CB\#3280, Chapel Hill, NC 27599, USA

3 McAllister Heart Institute, The University of North Carolina at Chapel Hill, Chapel Hill, NC 27599, USA

4 Department of Biological Sciences, University of Denver, Denver, CO, USA
Lumenization closely follows sprout formation and extension temporally, and many sprout tips have multiple EC that are polarized in the apical-basal axis [9]. However, it is not known how centrosome number contributes to this process.

As tumors grow, hypoxia leads to elevated levels of proangiogenic factors that promote neo-angiogenesis to vascularize the tumor. However, the blood vessels surrounding these tumors are often leaky, tortuous, and not properly lumenized [10-12], suggesting defects in EC junctions and polarity. Interestingly, tumor vessels are also characterized by EC with excess centrosomes [13,14]. The centrosome acts as a major microtubule organizing center and determinant of cell polarity $[15,16]$. High VEGF-A signaling in EC results in centrosome overduplication, which affects interphase cells by increasing invasiveness, elevating microtubule nucleations, changing aspects of polarity and migration, and altering signaling dynamics [13, 17-19]. However, it is unclear how excess centrosomes impact cell-cell interactions and lumenogenesis.

Cadherins form cell-cell adherens junctions that link to the actin cytoskeleton and restrict the apical vs. basolateral domains [20, 21]. In EC, VE-cadherin is the primary component of the vascular adherens junction and is required for localization of apical markers during lumen formation in 
zebrafish [7]. Depletion of VE-cadherin is embryonically lethal in zebrafish and mice due to severe vascular defects, including a lack of vascular lumens [22, 23]. However, the effects of centrosome number on adherens junctions have not been investigated.

Here, we show for the first time that excess centrosomes prevent the proper polarization of interacting EC. Excess centrosomes resulted in EC with destabilized adherens junctions and narrow and closed vascular lumens both in vitro and in vivo. These findings reveal a novel role for the centrosome in EC apical-basal polarity, and in junction and lumen formation, and suggest how excess centrosomes in the vasculature may contribute to poor perfusion in tumor vessels.

\section{Results}

\section{Excess centrosomes perturb polarization of junctionally linked EC}

We previously showed that excess centrosomes interfere with repolarization along the forward-rearward axis in sprouting EC [17], and thus we hypothesized that supernumerary centrosomes disrupt multiple EC polarity axes. We examined the polarity between EC sharing junctions using an inducible system to overexpress polo-like kinase 4 (Plk4) in human umbilical vein endothelial cells (HUVEC) (Fig. 1a). Plk4 regulates centriole duplication, and its overexpression downstream of a TRE (tet-responsive element) upon addition of doxycycline (DOX) results in supernumerary centrosomes [13, 17, 24, 25]. TRE-Plk4 HUVEC were seeded onto large $\mathrm{H}$-shaped micropatterns that allow for polarity assessment between two EC that form a cell-cell junction. Polarity was defined based on centrosome position relative to the nucleus and cell-cell junction, with a "proximal" position being near the cell-cell junction, a "central" position in the nuclear region, and a "distal" position between the nucleus and the cell periphery (Fig. 1b). Two EC with a normal centrosome number (1-2) typically had a distal centrosome polarity (Fig. 1c-d). In contrast, two EC with excess centrosomes $(>2)$ showed a significant increase in central/ proximal polarity, indicating that centrosome number affects EC polarization relative to cell-cell junctions (Fig. 1c, d). Interestingly, when a normal EC and an EC with excess centrosomes were linked on the same pattern $(\mathrm{N}: \mathrm{O})$, normal EC had a higher frequency of aberrant centrosome localization
Fig. 1 Excess centrosomes perturb polarity between EC sharing junctions.

a Schematic of Plk4 overexpression in HUVEC. TRE, tet-responsive element; rtTA, reverse tet transactivator. b Schematic of H-micropattern with possible centrosome positions. c Representative images of EC on H-micropatterns with either 1-2 centrosomes (left) or $>2$ centrosomes (right). Yellow arrowheads, 1-2 centrosomes with distal localization; white arrowheads, $>2$ centrosomes with central localization; centrosome ( $\gamma$-tubulin, green); DNA (blue, DRAQ7). Insets, $\gamma$-tubulin. Scale bar, $10 \mu \mathrm{m}$. d Quantification of EC centrosome position based on pattern orientation and $\mathrm{EC}$ with 1-2 centrosomes $(\mathrm{N})$ or $>2$ centrosomes $(\mathrm{O})$. e Quantification of polarity based on pattern orientation. f Quantification of polarity mismatch. $n=4$ replicates. Statistics: $\chi^{2}$ analysis. (N:N), both EC 1-2 centrosomes/cell; (O:O), both EC $>2$ centrosomes/cell; ( $\mathrm{N}: \mathrm{O})$ one EC 1-2 centrosomes, one EC $>2$ centrosomes
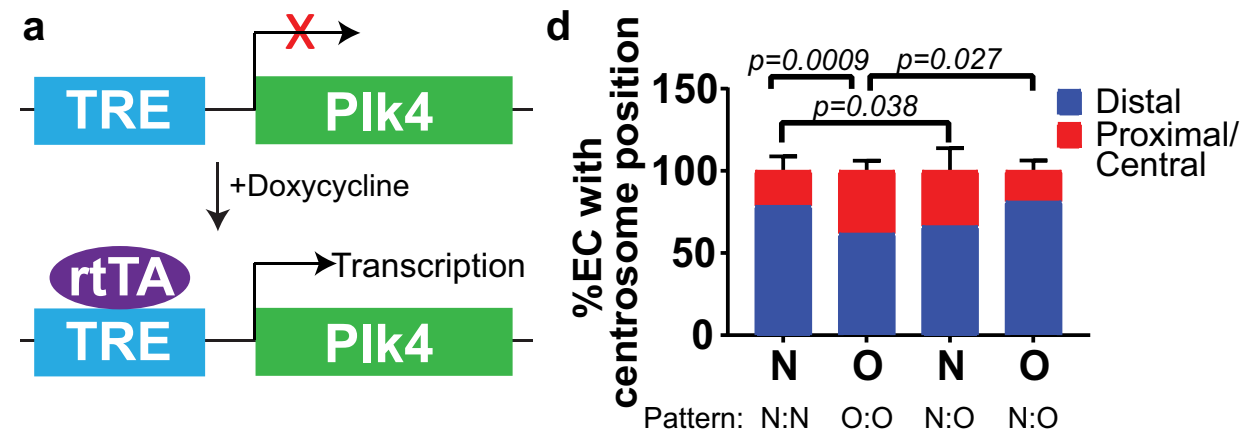

b
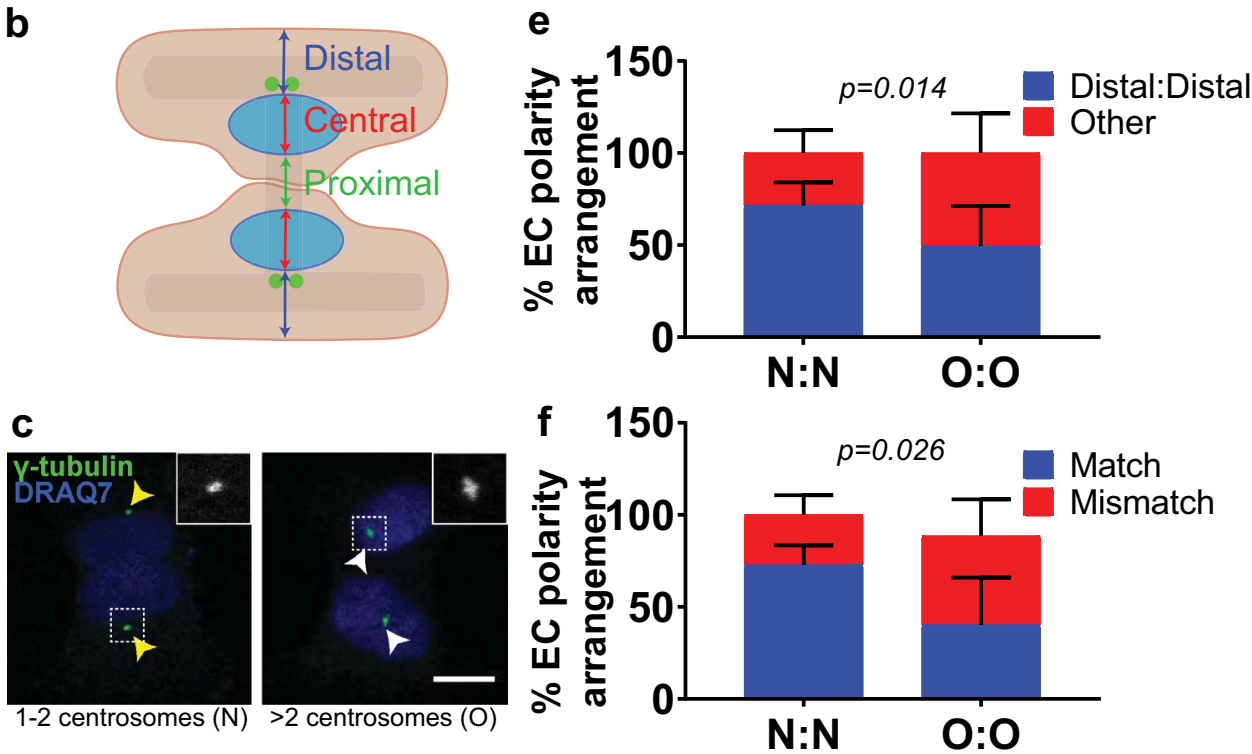
than $\mathrm{N}: \mathrm{N}$ counterparts, while $\mathrm{EC}$ with excess centrosomes had a higher frequency of normal centrosome localization relative to $\mathrm{O}: \mathrm{O}$ counterparts, suggesting that EC polarity influences polarity in neighboring cells (Fig. 1d). Furthermore, when two EC with normal centrosome number $(\mathrm{N}: \mathrm{N})$ formed a junction, both cells usually had centrosomes in the distal position, which we termed "distal" polarity (Fig. 1e). All other polarity combinations were deemed "other". When both EC forming a junction had excess centrosomes (O:O), polarity was randomized between distal and other (Fig. 1e). Additionally, N:N EC combinations were more likely to have cells with matching polarity, while EC with an O:O combination were less likely to have the same polarity as their neighbor (Fig. 1f). Taken together, these data indicate that excess centrosomes prevent proper EC polarization relative to cell-cell junctions.

\section{EC with excess centrosomes form disorganized adherens junctions}

Because interacting EC with excess centrosomes had perturbed polarity in the junction axis, we asked whether EC cell-cell interactions were influenced by centrosome number. Adherens junctions are required for proper apical-basal polarity in epithelial cells [7, 26], and the distribution of VEcadherin at EC adherens junctions is indicative of junction maturity; a linear VE-cadherin pattern is associated with mature stabilized junctions, while a serrated and punctate VE-cadherin pattern is associated with activated and/or disrupted junctions [27]. To determine how excess centrosomes influence adherens junctions, we seeded induced TRE-Plk4 HUVEC onto H-shaped micropatterns. Due to the heterogeneous nature of Plk4-induced centrosome overduplication, a given cell-cell junction could have contributions from two EC with a normal number of centrosomes $(\mathrm{N}: \mathrm{N})$, two EC with excess centrosomes $(\mathrm{O}: \mathrm{O})$, or one of each $(\mathrm{N}: \mathrm{O})$ (Fig. 2a-a"). We found that junctions with contributions from EC with normal centrosome numbers had more linear and stable junctions, while junctions with contributions from $\mathrm{EC}$ with excess centrosomes formed more chaotic and disrupted junctions (Fig. 2b-b", c-c"). These chaotic junctions had increased total VE-cadherin area (Fig. 2d), and line scans revealed a more dispersed VE-cadherin junction distribution when one or both EC had excess centrosomes
Fig. 2 EC with excess centrosomes form disorganized adherens junctions.

a-a" Schematics showing potential arrangement of $\mathrm{EC}$ on $\mathrm{H}$ patterns. b-b"' Representative images of cell-cell junctions on $\mathrm{H}$ patterns with $\mathrm{EC}$ centrosome status $\mathrm{N}: \mathrm{N}(\mathbf{b}), \mathrm{N}: \mathrm{O}\left(\mathbf{b}^{\prime}\right)$, or $\mathrm{O}: \mathrm{O}(\mathbf{b}$ '). EC were stained for $\gamma$-tubulin (green, centrosome), VE-cadherin (red, adherens junctions), and DRAQ7 (blue, DNA). Scale bar, $10 \mu \mathrm{m}$. (c-c',) Representative VE-cadherin line scans between centrosomes in $\mathrm{N}: \mathrm{N}(\mathbf{c}), \mathrm{N}: \mathrm{O}\left(\mathbf{c}^{\prime}\right)$, or $\mathrm{O}: \mathrm{O}$ (c") EC junctions. \#, positive peak. d Quantification of VEcadherin area in EC junctions with the indicated centrosome complements. e Quantification of peaks/length of VE-cadherin line scans of EC junctions with indicated centrosome complements. f Quantification of junction width of EC with indicated centrosome complements. $n=4$ replicates. Statistics: one-way ANOVA with Tukey's correction. *, $p \leq .05$, **, $p \leq .01$, ***, $p \leq .001, * * * *, p \leq .0001$
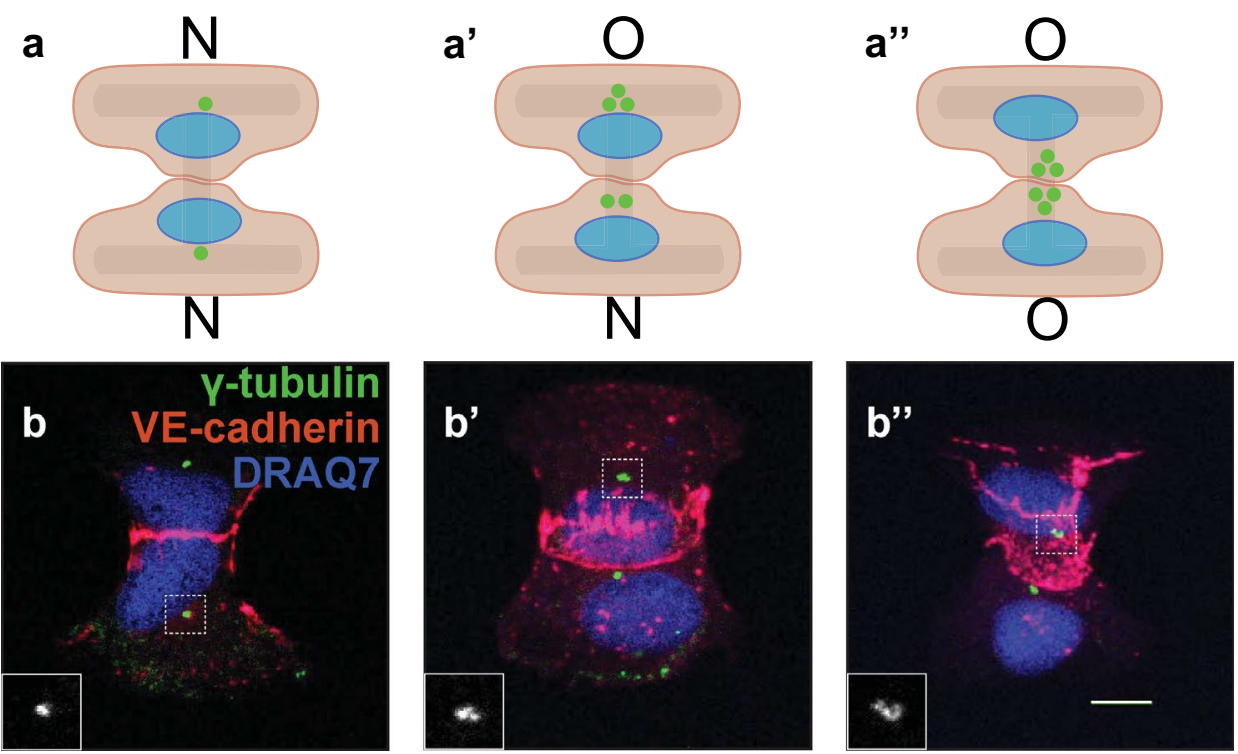

C

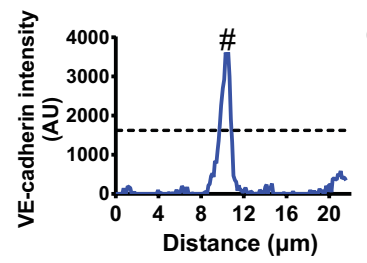

d

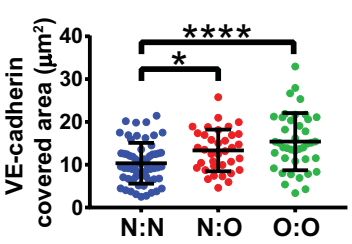

c'

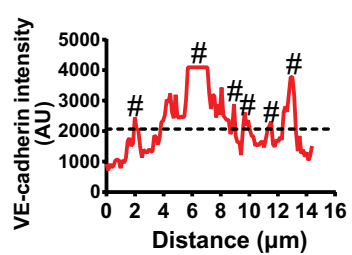

e

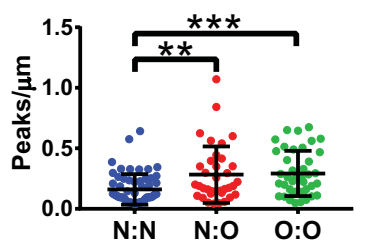

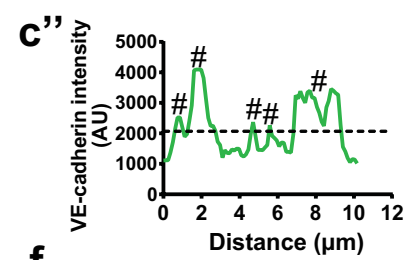

$f$

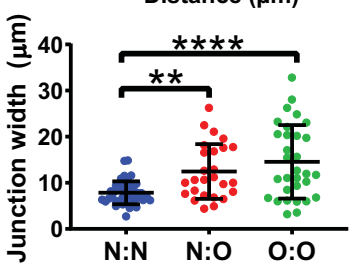


(Fig. 2e). We also assessed the overall width of the junction and found that junctional VE-cadherin distribution was wider in junctions formed when one or both EC had excess centrosomes (Fig. 2f). Together, these data suggest that both the junction shape and VE-cadherin distribution are perturbed in EC with supernumerary centrosomes. Because tight junctions are maintained along with ectopic patches of VE-cadherin in migrating and anastomosing EC [28], we examined tight junctions via ZO-1 staining and found that both $\mathrm{N}: \mathrm{O}$ and $\mathrm{O}: \mathrm{O}$ patterns had missing or abnormal tight junctions downstream of disrupted adherens junctions, with $\mathrm{O}: \mathrm{O}$ patterns having a more severe disruption (Fig. S1). In these cases, there was no organized ZO-1 staining in the large patches of VE-cadherin beyond the junction site. Overall, these findings indicate that EC with excess centrosomes do not maintain proper endothelial cell-cell adherens or tight junctions.

\section{EC with excess centrosomes have abnormal lumens}

We hypothesized that the disrupted polarity seen between EC with supernumerary centrosomes had physiological consequences. To further examine EC polarity, we analyzed lumenized sprouts that form and polarize in the apical-basal axis in a $3 \mathrm{D}$ sprouting angiogenesis assay [9, 29]. DOXtreated TRE-Plk4 HUVEC expressing centrin::eGFP were stained for Moesin 1 as an apical marker, since Moesin 1 localizes to F-actin and is typically found at apical EC cell-cell contacts [6] (Fig. 3a, b). We found that EC with normal centrosome numbers (1-2) had apically positioned centrosomes, while EC with excess centrosomes $(>2)$ had randomized centrosome polarity in the apical-basal axis (Fig. 3b, c). EC with excess centrosomes were more likely to have punctate Moesin1, basal Moesin1, or unpolarized abnormal Moesin1 staining patterns compared to normal linear and apical patterns (Fig. 3b, d), indicative of polarity defects. Finally, VE-cadherin around EC with excess centrosomes was more dispersed in 3D sprouts compared to EC with normal centrosome number (Fig. S2a).

Since both apical-basal polarity and proper junction formation are important for lumen formation [4-8], we hypothesized that excess centrosomes in EC perturb vessel lumenogenesis. After normalizing for overall vessel width, vessel lumen widths were narrower near EC with excess centrosomes (excluding closed lumens) compared to EC with normal centrosome numbers (Fig. 3b, e), and raw lumen widths were also decreased (Fig. S2b). Interestingly, some lumens were completely closed around EC with
Fig. 3 Sprouts containing EC with excess centrosomes have abnormal lumens.

a Schematic showing the XY plane of vessel sprout and centrosome position scoring criteria. b Representative images showing lumen diameter in EC with indicated centrosome number. Left panels, $\mathrm{XY}$ axis; right panels, $\mathrm{YZ}$ axis. Centrin::GFP (green, centrosome), Moesin1 (red, apical), DNA (cyan, DAPI). Insets, centrin-GFP. Scale bar, $10 \mu \mathrm{m}$. c Apical-basal centrosome position in sprouting EC with indicated centrosome number. $n=3$ replicates. Statistics: $\chi^{2}$ analysis. d Moesin1 staining pattern in EC with indicated centrosome numbers. $n=3$ replicates. Statistics: $\chi^{2}$ analysis. e Quantification of lumen diameter in EC with indicated centrosome number. $n=3$ replicates. Statistics: one-way ANOVA with Tukey's correction. **, $p \leq .01$. f Quantification of open, narrow, or closed lumens in EC with indicated centrosome number. $n=3$ replicates. Statistics: $\chi^{2}$ analysis a
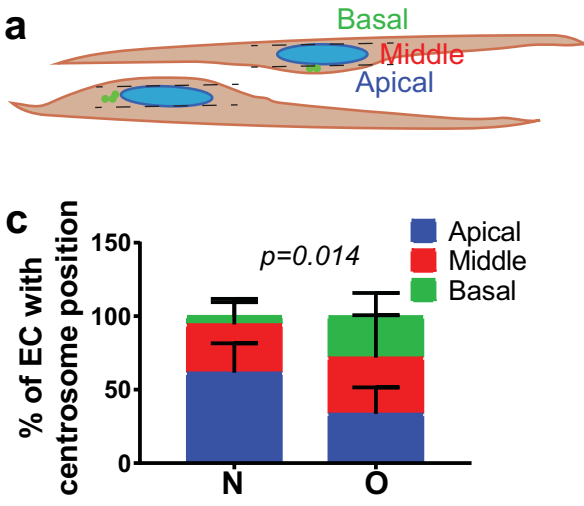

d

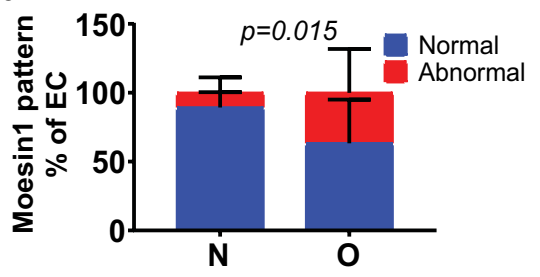

e

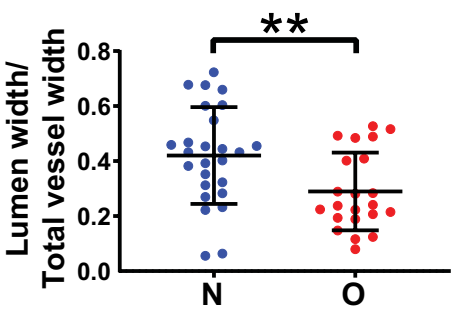

b
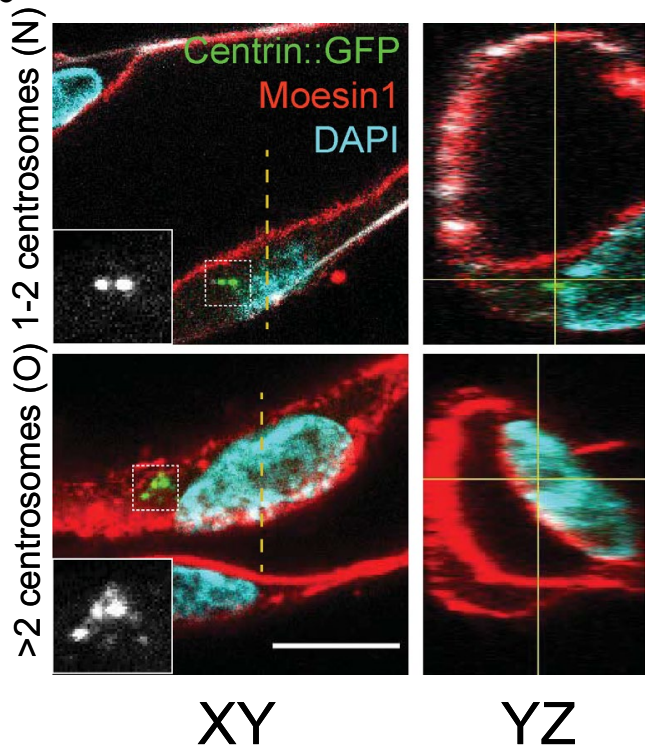

$\mathrm{YZ}$
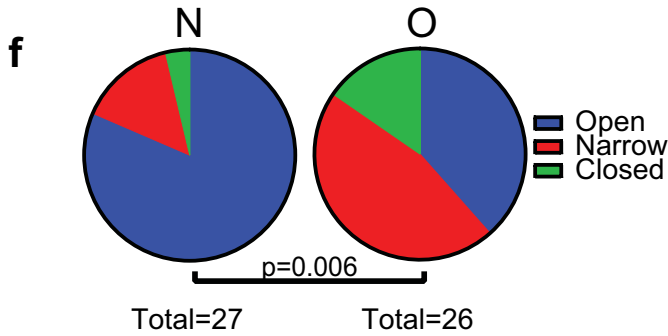
supernumerary centrosomes, and the incidence of closed and narrowed lumens was elevated near sprouting EC with excess centrosomes (Fig. 3f). These findings indicate that EC centrosome number regulates proper vessel lumen diameter, likely through effects on apical-basal polarity.

\section{Overexpression of Plk4 in zebrafish results in fewer lumenized vessels}

To determine if centrosome number affects lumen formation in vivo, we utilized a zebrafish model with vascularspecific Cre-mediated overexpression of Plk4 (Fig. S3a), and crossed to $T g$ (fli:centrin-GFP) fish to visualize EC centrosomes. Fish overexpressing Plk4 had increased EC with $>2$ centrosomes (Fig. S3b-c), and microangiography at 72 hpf revealed that these fish also had fewer perfused intersegmental vessels (ISVs) compared to wild type controls or fish lacking Cre (Fig. 4a, b). Lumens of perfused vessels in fish with Plk4 overexpression were narrower compared to controls, and more lumens were completely closed or narrowed (Fig. 4c, d). To further test the effects of supernumerary centrosomes, we generated a second zebrafish model by utilizing the Gal4-UAS system to transiently overexpress Plk4 in blood vessels (Fig. S3d). ISVs containing EC with excess
Fig. 4 Plk4 overexpression in zebrafish results in fewer lumenized vessels.

a-a" Representative images of zebrafish tails at $72 \mathrm{hpf}$ perfused with Qdot605 to assess lumen formation in embryos of indicated genotype and indicated injection with Cre.

Blue arrowheads, open lumens; red arrowheads, narrow lumens; green arrowheads, closed lumens. Insets, examples of different lumen categories. LSL, lox-STOP-lox. b Quantification of ISV perfusion in $72 \mathrm{hpf}$ fish with indicated genotypes/ condition (WT + Cre, $n=5$ fish; LSL-Plk4 uninjected, $n$ $=31$; LSL-Plk4 + Cre, $n=28$ ). c Quantification of lumen width in $72 \mathrm{hpf}$ fish with indicated genotypes/condition (WT + Cre, $n=86$ lumens; LSL-Plk4 uninjected, $n=628$; LSLPlk4+Cre, $n=450)$. Statistics: one-way ANOVA with Tukey's correction. *, $p \leq .05, * * * *$, $p \leq .0001$. d Quantification of open, narrow, or closed lumens in vessels of $72 \mathrm{hpf}$ fish with indicated genotypes/condition. $N=5$ fish/condition. Statistics: $\chi^{2}$ analysis. e Representative images of ISVs from $72 \mathrm{hpf}$ embryos characterized by presence of $\mathrm{Tg}(\mathrm{fli}: \mathrm{Gal} 4)$ and centrosome number. $T g$ ( $k d r l: m C h e r r y)$ (red), $T$ (fli:centrin-GFP) (green). Insets, centrin-GFP. Scale bar, $20 \mu \mathrm{m}$. f Quantification of ISV width with indicated genotypes/condition (Fli:Gal4 negative, $n=4$ fish; Fli:Gal4 positive, $n=13$ ). Statistics: one-way ANOVA with Tukey's correction. ****, $p \leq .0001$
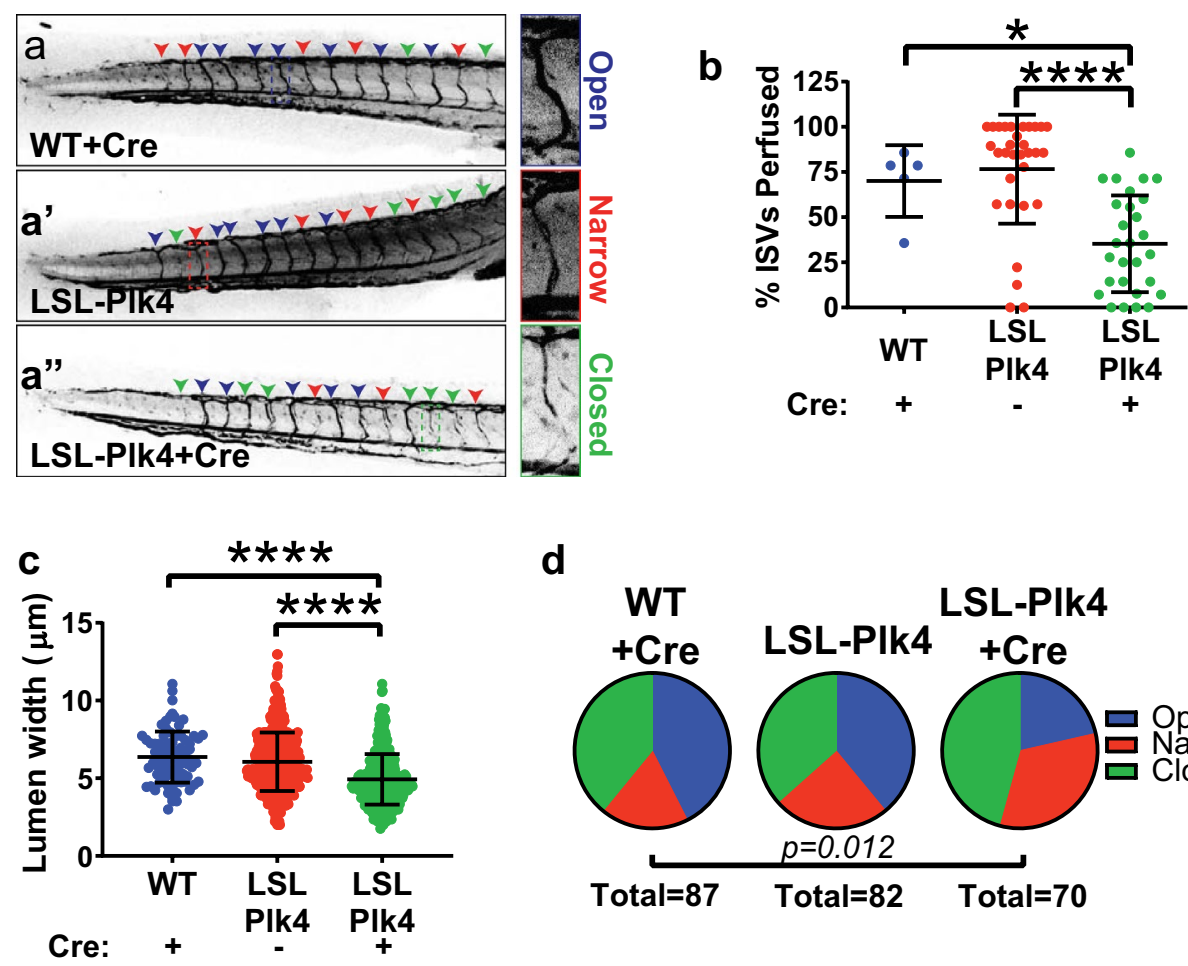

d
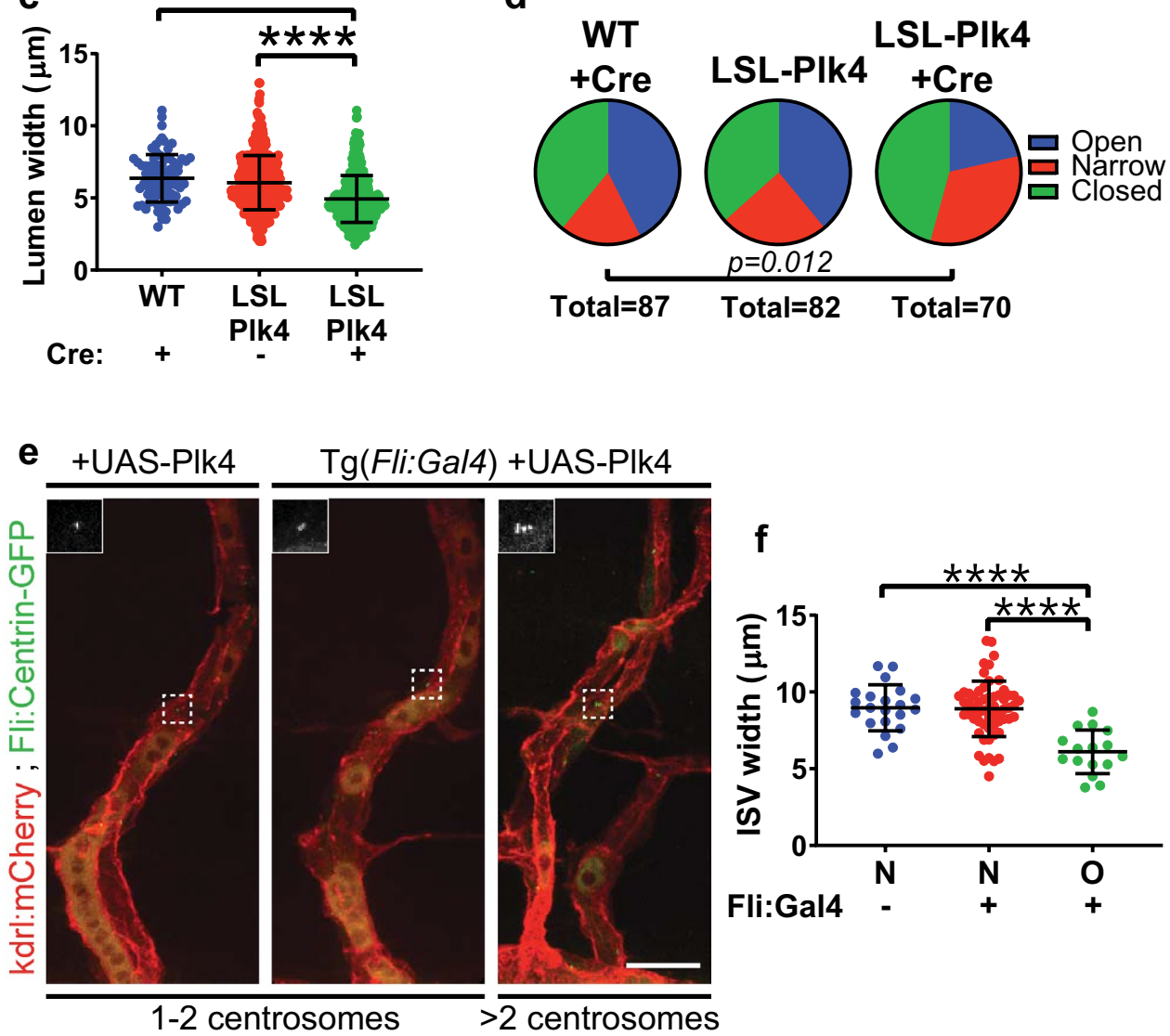
centrosomes were narrower than neighboring ISVs consisting of EC with 1-2 centrosomes, and ISVs from fish lacking $\mathrm{Tg}$ (fli:Gal4) (Fig. 4e, f). These findings indicate that ISV narrowing is specific to sprout regions with EC harboring excess centrosomes. Reduced vessel perfusion, coupled with narrow or blocked lumens and vessels, suggests that excess centrosomes in $\mathrm{EC}$ lead to disrupted lumenogenesis in vivo.

\section{Discussion}

Angiogenesis involves migration and rearrangement of EC, and leads to new conduits through anastomosis and lumen formation between adjacent EC [1-3]. Cell-cell interactions and polarization in several axes are important for lumen formation, but the role of the centrosome in these processes is poorly understood. Here, we show that supernumerary centrosomes perturb EC polarity and cell adhesion and disturb lumen formation in vitro and in vivo, implying that the centrosome is involved in both junctional adhesion and the apical-basal polarization that precedes vascular lumen formation or stabilization.

Our data highlight important roles for the centrosome in maintaining junction integrity and lumen development in interphase cells. Besides overduplication, centrosome function is impacted by centriole loss or structural defects. Mislocalization of centrosomal proteins, structural deficits, and centrosome loss all result in G1 cell-cycle arrest [30], and compromise analysis of effects of centrosome loss on interphase cell behavior. Although supernumerary centrosomes affect cellular processes, they do not acutely block cell-cycle progression [17] and thus allow for analysis of centrosome perturbation on interphase behaviors.

Our previous work showed that EC centrosome overduplication is linked to microtubule defects, with increased nucleations and catastrophes accompanied by forward-rearward polarity defects [17]. Changes in microtubule dynamics are linked to regulation of adherens junctions in EC [31]. Our data indicate that adherens junctions are significantly disrupted in EC with excess centrosomes, suggesting that junctions are disrupted downstream of centrosome-induced microtubule defects.

Lumen structure is also disrupted downstream of excess centrosomes in sprouting EC, suggesting an apical-basal polarity defect. It is likely that junctional defects contribute to poor lumenization in vitro and in vivo. VE-cadherin at EC junctions is linked to vascular lumen formation during anastomosis [32, 33]. Moreover, genetic loss of VE-cadherin in mice or zebrafish leads to lumen defects, vascular dysfunction, and embryonic lethality [22, 23], supporting a link between disrupted adherens junctions and malformed lumens in EC sprouts. Proper adherens junctions are necessary to stabilize tight junctions and form lumens [34, 35], and here we show that tight junction formation is also compromised in EC with excess centrosomes, suggesting that tight junction defects contribute to lumen defects.

We show that lumens are perturbed following centrosome overduplication in both an in vitro angiogenesis model lacking physiological flow and in vivo, in zebrafish with blood flow. These findings indicate that the lumenal defects resulting from excess centrosomes do not depend on shear stress exerted by blood flow. While lumenization requires blood flow during late stages of angiogenesis [36], lumens also form in vitro and in vivo absent blood flow [7, 37]. Disruption of the VE-cadherin junctional complex is sufficient to prevent luminal expansion independent of blood flow through changes in cell contractility [37]; thus, it is likely that the profound changes in VE-cadherin localization at EC cell-cell junctions following centrosome overduplication alter the ability of EC to properly coordinate the cytoskeletal forces needed for polarization and lumenogenesis.

Centrosome amplification is a common phenomenon in tumors and has been linked to changes in the tumor microenvironment, including endothelial cells [13, 14]. Local hypoxia in tumors increases VEGF-A signaling, leading to high CyclinE/cdk2 that promotes EC centrosome overduplication [19], and elevated Plk4 expression occurs in human and mouse tumor vessels [38, 39]. Although therapies targeting angiogenesis have had mixed results [40, 41], understanding tumor $\mathrm{EC}$ responses is necessary for design of more complex multi-modal anti-tumor therapies [41-44]. In summary, by revealing a unique link between centrosome perturbation and adherens junction stability and lumenization, our data indicate a novel role for the centrosome in EC polarity, junctions, and lumenization in blood vessels. These abnormalities may contribute to tumor blood vessel dysfunction.

\section{Materials and methods}

\section{Cell culture}

HUVEC (C2519A, lot 486,264, Lonza) were cultured in EBM-2 (CC-3162, Lonza) supplemented with the Endothelial Growth Medium (EGM-2) bullet kit (CC-3162, Lonza) and $1 \mathrm{x}$ antibiotic-antimycotic (Gibco). Normal human lung fibroblasts (CC2512, Lonza) were cultured in DMEM (Gibco) with $10 \%$ fetal bovine serum (FBS) and 1x antibiotic-antimycotic (Gibco). All cells were maintained at $37^{\circ} \mathrm{C}$ and $5 \% \mathrm{CO}_{2}$. Tetracycline-inducible Plk4 overexpression HUVEC were generated as previously described [13, 17, 25]. Viruses were produced by the UNC Lenti-shRNA Core Facility. To induce centrosome overduplication, tetPlk4 HUVEC were treated with $1 \mu \mathrm{g} / \mathrm{ml}$ doxycycline (DOX, 
D9891, Sigma) for $24 \mathrm{~h}$ followed by washout. Experiments were performed $48 \mathrm{~h}$ following DOX addition.

\section{D sprouting angiogenesis assay}

The 3D sprouting angiogenesis assay was performed as previously described with the following changes [17, 45]. TRE-Plk4 HUVEC were treated with $1 \mu \mathrm{g} / \mathrm{ml}$ DOX for $24 \mathrm{~h}$ at $37^{\circ} \mathrm{C} .13 \mu \mathrm{l} / \mathrm{ml}$ centrin:eGFP lentivirus and $1 \mu \mathrm{g} / \mathrm{ml}$ Polybrene were added to HUVEC $16 \mathrm{~h}$ post DOX addition for the last $8 \mathrm{~h}$ of DOX treatment. Cells were washed with Dulbecco's phosphate-buffered saline (DPBS) (MT-21-031-CV, Corning) and fresh media was added. The following day, infected cells were coated onto cytodex 3 microcarrier beads (17,048,501, GE Healthcare Life Sciences) and embedded in a fibrin matrix as described. Z-stacks were acquired with an Olympus confocal microscope (FV1200 or FV3000) and processed in Image $\mathbf{J}$ (National Institutes of Health). Total vessel width was measured in the YZ plane by drawing a line through the nucleus and across the entire vessel. Lumen width was measured in the $\mathrm{YZ}$ plane using Moesin1 staining (1:1000, ab52490, Abcam) as a surrogate for the apical surface and normalized to total vessel width. Lumens were considered "narrow" if the normalized lumen width fell below the mean lumen width minus one standard deviation of the normal condition. Lumens were considered "closed" if the lumen width was zero. DAPI $(10,236,276,001$, Sigma) was used to visualize the nucleus, and VE-cadherin was used to visualize adherens junctions $(1: 1000,2500 \mathrm{~S}$, Cell Signaling).

\section{Micropatterns}

Large H-micropatterns (1600 $\mu \mathrm{m}^{2}$, CYTOO) were coated with $5 \mu \mathrm{g} / \mathrm{ml}$ fibronectin and seeded with HUVEC according to manufacturer's instructions. Cells were allowed to spread for $24 \mathrm{~h}$, then fixed with ice-cold methanol for $10 \mathrm{~min}$ at $4{ }^{\circ} \mathrm{C}$.

\section{Immunofluorescence and quantification}

Fixed HUVEC were blocked for $1 \mathrm{~h}$ at RT in blocking solution (5\% FBS, $2 \mathrm{X}$ antibiotic-antimycotic (Gibco), $0.1 \%$ sodium azide (s2002-100G, Sigma) in DPBS). Cells were incubated in primary antibody overnight at $4{ }^{\circ} \mathrm{C}$, then washed $3 \mathrm{X}$ for $5 \mathrm{~min}$ in DPBS. Secondary antibody and DRAQ7 (1:1000, ab109202, Abcam) were added for $1 \mathrm{~h}$ at RT followed by $3 \mathrm{X}$ washes for $10 \mathrm{~min}$ each in DPBS. Slides were mounted with coverslips using Prolong Diamond Antifade mounting medium (P36961, Life Technology) and sealed with nail polish. Primary and secondary antibodies were diluted in blocking solution. Images were acquired with an Olympus confocal microscope (FV1200 or FV3000) and analyzed using ImageJ. Centrosome overduplication was quantified by counting centrosome number in at least 500 cells per replicate. Junction area on micropatterns was quantified by thresholding VE-cadherin staining and measuring total area within the junction in ImageJ. VE-cadherin distribution on doublet micropatterns was measured in ImageJ by drawing a line across the junction (from centrosome in first cell to centrosome in second cell) and acquiring a line scan of VE-cadherin intensity. Peaks were counted for each pattern and normalized to the line length. Only peaks above half of the maximum VE-cadherin intensity were counted. Junction width was measured by creating a histogram of the VE-cadherin signal integrated over the y-axis and measuring the width of the most prominent peak(s). The following primary antibodies were used: anti- $\gamma$-tubulin (1:5000, T6557, Sigma-Aldrich), anti-VE-cadherin (1:500, 2500S, Cell Signaling), anti-ZO-1 (1:500, MABT339, Millipore Sigma), anti-Moesin-1 (1:1000, ab52490, Abcam). The following secondary antibodies from Life Technologies were used: goat anti-mouse AlexaFluor 488 (1:500, A11029), goat anti-mouse AlexaFluor 594 (1:500, A11005), goat anti-mouse AlexaFluor 647 (1:500, A21236) goat anti-rabbit AlexaFluor 488 (1:500, A11034), goat anti-rabbit AlexaFluor 594 (1:500, A11037).

\section{Zebrafish}

Zebrafish (Danio rerio) were housed in an institutional animal care and use committee (IACUC)-approved facility and maintained as previously described (Wiley et al. 2011). $T g(k d r l: m C h e r r y)$ was a gift from D. Stainier. $T g(f l i: G a l 4)$ was a gift from W. Herzog. $T g(f i$ :centrin-GFP) was created by subcloning centrin:GFP into the $\mathrm{pCR} 8 / \mathrm{GW} / \mathrm{TOPO}$ middle entry vector (Thermo Fisher) and then gateway cloning into the pDestTol2CG2 plasmid (REF Tol2 kit). Tol2 transposase RNA was generated using the sp6 mMessage mMachine synthesis kit (AM1340, Thermo Fisher). For overexpression of Plk4, p2a-Lox-mCherry-STOP-LoxPlk4 was fused to the 3' end of the endogenous kdrl gene. The assembled construct was subcloned into the pKHR4 backbone and linearized by I-SceI digest. This construct, $200 \mathrm{ng} / \mu \mathrm{l}$ sgRNA (5'-TCTGGTTTGGAAGGACACAG-3'), and $700 \mathrm{ng} / \mu \mathrm{l}$ of Cas9 recombinant protein (PNABio) were injected into one-cell stage $T g(f i$ :centrin-GFP) embryos to generate double stranded breaks at the 3 ' end of the $k d r l$ gene and induce homologous recombination as previous described [46]. F1 fish were injected with Cre mRNA at the 1-2 cell stage. Cre mRNA was obtained by subcloning the $\mathrm{Cre}$ gene from the pME-ERT2-Cre-ERT2 plasmid into the $\mathrm{pCR} 8 / \mathrm{GW} / \mathrm{TOPO}$ middle entry vector, gateway cloning into a pCS Dest vector, then preparing RNA with the sp6 mMessageMachine synthesis kit. pME-ERT2-CreERT2 was a gift from Kryn Stankunas (Addgene plasmid 
\# 82,587; http://n2t.net/addgene:82587; RRID:Addgene 82,587). Embryos were collected from desired crosses and grown in E3 at $28.5^{\circ} \mathrm{C}$. At $72 \mathrm{hpf}$, embryos were injected with Qdot605 (Q10103MP, Thermo Fisher). Plk4 overexpression was determined by the loss of mCherry signal.

A second fish model was generated as follows: a $5 X$-UAS-Plk4 construct was generated using Gibson cloning by fusing a $5 X-U A S$ tag upstream of zebrafish Plk4. pUAS:Cas9T2AGFP;U6:sgRNA1;U6:sgRNA2 was a gift from Filippo Del Bene (Addgene plasmid \#74,009; http:// n2t.net/addgene:74009;RRID:Addgene_74009) [47]. This construct and I-SceI meganuclease were injected into onecell fish from crosses between $T g(f i$ :centrin-GFP) and $\mathrm{Tg}$ (fli:Gal4;kdrl:mCherry), and fish were grown in E3 at $28.5^{\circ} \mathrm{C}$ to $72 \mathrm{hpf}$. Fish were fixed by incubating dechorionated embryos in ice-cold $4 \%$ PFA at $4{ }^{\circ} \mathrm{C}$ overnight. Embryos were rinsed in PBS and mounted using a fine probe to de-yolk and a small blade to separate the trunk from the cephalic region, and mounting with VECTASHIELD ${ }^{\circledR}$ Hardset ${ }^{\text {TM }}$ Antifade Mounting Medium. The coverslip was sealed with petroleum jelly before imaging on an Olympus FV1200 confocal microscope. Centrosome overduplication was confirmed based on centrinGFP centriole labeling. ISV perfusion was measured by counting the number of perfused ISVs based on Qdot605 signal as a percentage of total ISV number (visualized using $T g(k d r l: m C h e r r y))$. Lumen width was measured by measuring the diameter of perfused ISVs near the junction with the dorsal aorta. Lumens were considered "narrow" if the lumen width fell below the mean lumen width minus one standard deviation of the WT + Cre condition. Lumens were considered closed if the ISV perfusion was discontinuous.

\section{Statistics}

Student's two-tailed $t$ test was used to determine statistical significance in experiments with two groups. One-way ANOVA with Tukey's multiple comparisons test was used to determine statistical significance for experiments with 3 groups, and $\mathrm{X}^{2}$ was used for categorical data. Error bars represent the mean \pm standard deviation. Statistical tests and graphs were made using the Prism 7 software (GraphPad Software).

Acknowledgements We thank Tony Perdue and the UNC Biology Microscopy Core; the UNC Lenti-shRNA Core; Maryanna Parker, Joshua Blair, and Kaitlyn Quigley for fish room support; and Bautch lab members for critical discussions.

We recently became aware of a paper (Ricolo et al. (2016) Centrosome amplification increases single-cell branching in post-mitotic cells. Curr Biol 26:2805-2813) describing effects of centrosome manipulation in Drosophila trachea cells, including effects of centrosome loss on lumen formation.

Funding This work was supported by grants from the National Institutes of Health (HL43174, HL116719, HL117256, HL139950 to VLB), a K99/R00 (1K99HL124311-01A1 to EJK), the Integrated Vascular Biology Training Grant (5T32HL069768-17 to DBB), and an American Heart Association Predoctoral Fellowship (19PRE34380887 to DBB).

\section{Compliance with ethical standards}

Conflict of interest All authors declare that they have no conflict of interest.

Open Access This article is licensed under a Creative Commons Attribution 4.0 International License, which permits use, sharing, adaptation, distribution and reproduction in any medium or format, as long as you give appropriate credit to the original author(s) and the source, provide a link to the Creative Commons licence, and indicate if changes were made. The images or other third party material in this article are included in the article's Creative Commons licence, unless indicated otherwise in a credit line to the material. If material is not included in the article's Creative Commons licence and your intended use is not permitted by statutory regulation or exceeds the permitted use, you will need to obtain permission directly from the copyright holder. To view a copy of this licence, visit http://creativecommons.org/licenses/by/4.0/.

\section{References}

1. Carmeliet P, Jain RK (2011) Molecular mechanisms and clinical applications of angiogenesis. Nature 473(7347):298-307

2. Bautch VL, Caron KM (2015) Blood and lymphatic vessel formation. Cold Spring Harb Perspect Biol 7(3):a008268

3. Kushner E, Bautch V (2013) Building blood vessels in development and disease. Curr Opin Hematol 20(3):231-236

4. Blum Y, Belting HG, Ellertsdottir E, Herwig L, Luders F, Affolter M (2008) Complex cell rearrangements during intersegmental vessel sprouting and vessel fusion in the zebrafish embryo. Dev Biol 316:312-322

5. Iruela-Arispe ML, Davis GE (2009) Cellular and molecular mechanisms of vascular lumen formation. Dev Cell 16(2):222-231

6. Strilic B, Kucera T, Eglinger J, Hughes MR, McNagny KM, Tsukita $S$ et al (2009) The molecular basis of vascular lumen formation in the developing mouse aorta. Dev Cell 17(4):505-515

7. Wang Y, Kaiser MS, Larson JD, Nasevicius A, Clark KJ, Wadman SA et al (2010) Moesin1 and VE-cadherin are required in endothelial cells during in vivo tubulogenesis. Development 137(18):3119-3128

8. Lee CY, Bautch VL (2011) Ups and downs of guided vessel sprouting: the role of polarity. Physiology 26(5):326-333

9. Pelton JC, Wright CE, Leitges M, Bautch VL (2014) Multiple endothelial cells constitute the tip of developing blood vessels and polarize to promote lumen formation. Development 141(21):4121-4126

10. Aird WC (2012) Endothelial cell heterogeneity. Cold Spring Harb Perspect Med 2(1):a006429

11. Hashizume H, Baluk P, Morikawa S, McLean JW, Thurston G, Roberge $S$ et al (2000) Openings between defective endothelial cells explain tumor vessel leakiness. Am J Pathol 156(4):1363-1380 
12. Carmeliet P, Jain RK (2011) Principles and mechanisms of vessel normalization for cancer and other angiogenic diseases. Nat Rev Drug Discov 10:417-427

13. Kushner EJ, Ferro LS, Liu J-Y, Durrant JR, Rogers SL, Dudley AC et al (2014) Excess centrosomes disrupt endothelial migration via centrosome scattering. J Cell Biol 206(2):257-272

14. Hida K, Hida Y, Amin DN, Flint AF, Panigrahy D, Morton CC et al (2004) Tumor-associated endothelial cells with cytogenetic abnormalities. Cancer Res 64(22):8249-8255

15. Kollman JM, Merdes A, Mourey L, Agard DA (2011) Microtubule nucleations by gamma-tubulin complexes. Nat Rev Mol Cell Biol 12:709-721

16. Bettencourt-Dias M, Glover DM (2007) Centrosome biogenesis and function: centrosomics brings new understanding. Nat Rev Mol Cell Biol 8:451-463

17. Kushner EJ, Ferro LS, Yu Z, Bautch VL (2016) Excess centrosomes perturb dynamic endothelial cell repolarization during blood vessel formation. Mol Biol Cell 27(12):1911-1920

18. Godinho SA, Picone R, Burute M, Dagher R, Su Y, Leung CT et al (2014) Oncogene-like induction of cellular invasion from centrosome amplification. Nature 510(7503):167-171

19. Taylor SM, Nevis KR, Park HL, Rogers GC, Rogers SL, Cook JG et al (2010) Angiogenic factor signaling regulates centrosome duplication in endothelial cells of developing blood vessels. Blood 116(16):3108-3117

20. Spracklen AJ, Peifer M (2015) Actin and apical constriction: some (re)-assembly required. Dev Cell 35(6):662-664

21. Nowotarski SH, Peifer M (2014) Cell biology: a tense but good day for actin at cell-cell junctions. Curr Biol 24(15):R688-R690

22. Carmeliet P, Lampugnani M-G, Moons L, Breviario F, Compernolle V, Bono F et al (1999) Targeted deficiency or cytosolic truncation of the VE-cadherin gene in mice impairs VEGF-mediated endothelial survival and angiogenesis. Cell 98(2):147-157

23. Montero-Balaguer M, Swirsding K, Orsenigo F, Cotelli F, Mione M, Dejana E (2009) Stable vascular connections and remodeling require full expression of VE-cadherin in zebrafish embryos. PLoS ONE 4(6):e5772

24. Habedanck R, Stierhof YD, Wilkinson CJ, Nigg EA (2005) The Polo kinase Plk4 functions in centriole duplication. Nat Cell Biol 7(11):1140-1146

25. Yu Z, Ruter DL, Kushner EJ, Bautch VL (2017) Excess centrosomes induce $\mathrm{p} 53$-dependent senescence without DNA damage in endothelial cells. FASEB J 10:4295-4304

26. Iden S, Rehder D, August B, Suzuki A, Wolburg-Buchholz K, Wolburg $\mathrm{H}$ et al (2006) A distinct PAR complex associates physically with VE-cadherin in vertebrate endothelial cells. EMBO Rep 7(12):1239-1246

27. Bentley K, Franco CA, Philippides A, Blanco R, Dierkes M, Gebala V et al (2014) The role of differential VE-cadherin dynamics in cell rearrangement during angiogenesis. Nat Cell Biol 16(4):309-321

28. Paatero I, Sauteur L, Lee M, Lagendijk AK, Heutschi D, Wiesner $\mathrm{C}$ et al (2018) Junction-based lamellipodia drive endothelial cell rearrangements in vivo via a VE-cadherin-F-actin based oscillatory cell-cell interaction. Nat Commun 9(1):3545

29. Lampugnani M-G, Orsenigo F, Rudini N, Maddaluno L, Boulday $\mathrm{G}$, Chapon $\mathrm{F}$ et al (2010) CCM1 regulates vascular-lumen organization by inducing endothelial polarity. J Cell Sci 123:1073-1080

30. Mikule K, Delaval B, Kaldis P, Jurcyzk A, Hergert P, Doxsey S (2007) Loss of centrosome integrity induces p38-p53-p21dependent G1-S arrest. Nat Cell Biol 9:160-170
31. Komarova YA, Huang F, Geyer M, Daneshjou N, Garcia A, Idalino L et al (2012) VE-cadherin signaling induces EB3 phosphorylation to suppress microtubule growth and assemble adherens junctions. Mol Cell 48(6):914-925

32. Lenard A, Ellertsdottir E, Herwig L, Krudewig A, Sauteur L, Belting H-G et al (2013) In vivo analysis reveals a highly stereotypic morphogenetic pathway of vascular anastomosis. Dev Cell 25(5):492-506

33. Herwig L, Blum Y, Krudewig A, Ellertsdottir E, Lenard A, Belting H-G et al (2011) Distinct cellular mechanisms of blood vessel fusion in the zebrafish embryo. Curr Biol 21(22):1942-1948

34. Campbell HK, Maiers JL, DeMali KA (2017) Interplay between tight junctions and adherens junctions. Exp Cell Res 358(1):39-44

35. Odenwald MA, Choi W, Buckley A, Shashikanth N, Joseph NE, Wang Y et al (2017) ZO-1 interactions with F-actin and occludin direct epithelial polarization and single lumen specification in 3D culture. J Cell Sci 130(1):243-259

36. Gebala V, Collins R, Geudens I, Phng L-K, Gerhardt H (2016) Blood flow drives lumen formation by inverse membrane blebbing during angiogenesis in vivo. Nat Cell Biol 18(4):443-450

37. Hultin S, Zheng Y, Mahdi M, Vertuani S, Gentili C, Balland M et al (2014) AmotL2 links VE-cadherin to contractile actin fibres necessary for aortic lumen expansion. Nat Commun 5:3743

38. Roudnicky F, Poyet C, Wild P, Krampitz S, Negrini F, Huggenberger R et al (2013) Endocan is upregulated on tumor vessels in invasive bladder cancer where it mediates VEGF-A-induced angiogenesis. Cancer Res 73(3):1097-1106

39. Goveia J, Rohlenova K, Taverna F, Treps L, Conradi L, Pircher A et al (2020) An integrated gene expression landscape profiling approach to identify lung tumor endothelial cell heterogeneity and angiogenic candidates. Cancer Cell 37(1):21-36

40. Brossa A, Buono L, Fallo S, Pla AF, Munaron L, Bussolati B (2019) Alternative strategies to inhibit tumor vascularization. Int J Mol Sci 20:6180

41. Tamura R, Tanaka T, Akasaki Y, Murayama Y, Yoshida K, Sasaki $H$ (2019) The role of vascular endothelial growth factor in the hypoxic and immunosuppressive tumor microenvironmnet: perspectives for therapeutic implications. Med Oncol 37(1):2

42. Sakurai Y, Akita H, Harashima H (2019) Targeting tumor endothelial cells with nanoparticles. Int J Mol Sci 20(23):5819

43. Zhao Y, Wang X (2019) Plk4: a promising target for cancer therapy. J Cancer Res Clin Oncol 145:2413-2422

44. Rivera-Rivera Y, Saavedra HI (2016) Centrosome - a promising anti-cancer target. Biologics 10:167-176

45. Nakatsu MN, Hughes CCW (2008) An optimized three-dimensional in vitro model for the analysis of angiogenesis. Methods Enzymol 443:65-82

46. Hoshijima K, Jurynec MJ, Grunwald DJ (2016) Precise editing of the zebrafish genome made simple and efficient. Dev Cell 36(6):654-667

47. Di Donato V, De Santis F, Auer TO, Testa N, Sánchez-Iranzo $\mathrm{H}$, Mercader N et al (2016) 2C-Cas9: a versatile tool for clonal analysis of gene function. Genome Res 26(5):681-692

Publisher's Note Springer Nature remains neutral with regard to jurisdictional claims in published maps and institutional affiliations. 